\title{
Modeling Photovoltaic Power
}

\author{
Fotis Mavromatakis \\ Department of Electrical Engineering \\ Technological Educational Institute \\ of Crete \\ Heraklion, Crete, Greece \\ fotis@staff.teicrete.gr,
}

\author{
Yannis Franghiadakis \\ Department of Electrical Engineering \\ Technological Educational Institute \\ of Crete \\ Heraklion, Crete, Greece \\ pvjfra@staff.teicrete.gr
}

\author{
Frank Vignola \\ Solar Radiation Monitoring \\ Laboratory \\ 1274 University of Oregon \\ Eugene, Oregon 97403-1274, USA \\ fev@uoregon.edu
}

\begin{abstract}
A robust and reliable model describing the power produced by a photovoltaic system is needed in order to be able to detect module failures, inverter malfunction, shadowing effects and other factors that may result to energy losses. In addition, a reliable model enables an investor to perform accurate estimates of the system energy production, payback times etc. The model utilizes the global irradiance reaching the plane of the photovoltaic modules since in almost all Photovoltaic (PV) facilities the beam and the diffuse solar irradiances are not recorded. The airmass, the angle of incidence and the efficiency drop due to low values of solar irradiance are taken into account. Currently, the model is validated through the use of high quality data available from the National Renewable Energy Laboratory (USA). The data were acquired with IV tracers while the meteorological conditions were also recorded. Several modules of different technologies were deployed but here we present results from a single crystalline module. The performance of the model is acceptable at a level of $5 \%$ despite the assumptions made. The dependence of the residuals upon solar irradiance temperature, airmass and angle of incidence is also explored and future work is described.
\end{abstract}

Keywords-Photovoltaic power; modeling; data analysis; spectral effects; low irradiance losses

\section{INTRODUCTION}

The monitoring of the performance of a PV system is important for the detection of possible problems during the operational life time of a photovoltaic system. Several models are available in the literature that provide estimates of the PV power based on a number of parameters [1]. In the approach used in this study, simple mathematical functions are utilized to describe the effects of the light traveling path through the atmosphere, angle of incidence, low irradiance and inverter efficiency [2,3]. Dust losses can be included if they are known. The above functions can be determined experimentally or adopted from the literature. A minimum number of input parameters was used to keep the approach as simple as possible while providing quality performance estimates. Despite the fact that during the NREL experiment all solar irradiance components were independently measured, the model incorporates only the global irradiance as this is actually measured in typical photovoltaic installations. The performance of the model is discussed along with the factors that affect it and how it can be improved.

\section{EXPERIMENTAL DATA}

\section{A. Maintaining the Integrity of the Specifications}

The model assumes that the solar irradiance affects the power production in two distinct ways. The first is the known linear dependence of photovoltaic power and the short circuit current, while the second involves the known reduction in efficiency ([3] and references there in). The cell temperature is assumed to be measured through the backside temperature. If it is not available, then it can be determined through measurements of the air temperature and the wind speed along with the global solar irradiance. However, the dependence of power on this term is much reduced due to the temperature coefficient of maximum power at STC conditions which is typical around $-0.4 \% / \mathrm{C}$ for crystalline modules. This coefficient is obtained from the manufacturer's data otherwise it can be determined from actual measurements. The angle of incidence effects for any given module can be determined through measurements of the short circuit current, the incident beam and diffuse irradiance, and the temperature of the module [4]. The way of performing such measurements is described in detail in [4]. Typically, such measurements are taken over a short period of time, e.g. around solar noon where the incoming solar radiation is not varying. It is then possible to separate the AOI effects from airmass/spectral effects. Currently this model assumes that the response of a module to the beam and diffuse irradiances is the same.

It is known that the nominal or peak power of a photovoltaic module is given at Standard Test Conditions (STC). These conditions assume a solar irradiance of 1000 $\mathrm{W} / \mathrm{m}^{2}$ at normal incidence, a cell temperature of 25 degrees Celsius and an air mass of 1.5 . However, it is clear that these conditions rarely hold outdoors, especially during the winter period in northern latitudes. The electromagnetic radiation coming from the sun is affected as a function of wavelength due to its propagation through the atmosphere. Rayleigh scattering is the dominant scattering mechanism under cloudless skies. This mechanism displays a strong wavelength dependence affecting mainly UV/optical wavelengths. It is evident that the module performance will also be affected. Since the spectral response of thin film modules lies mainly in the optical regime, these will be more affected than mono or polycrystalline modules. Air mass effects are studied through 
measurements of the short circuit current according to the method described in [4]. The data are reduced to STC conditions by correcting for the temperature and the instantaneous solar irradiance acquired during the measurements. Such measurements are especially useful since photovoltaic modules can be used to assess the spectrally averaged solar irradiance incident upon the module. The dependence of the produced DC power upon the air mass is examined through this function to a first approximation.

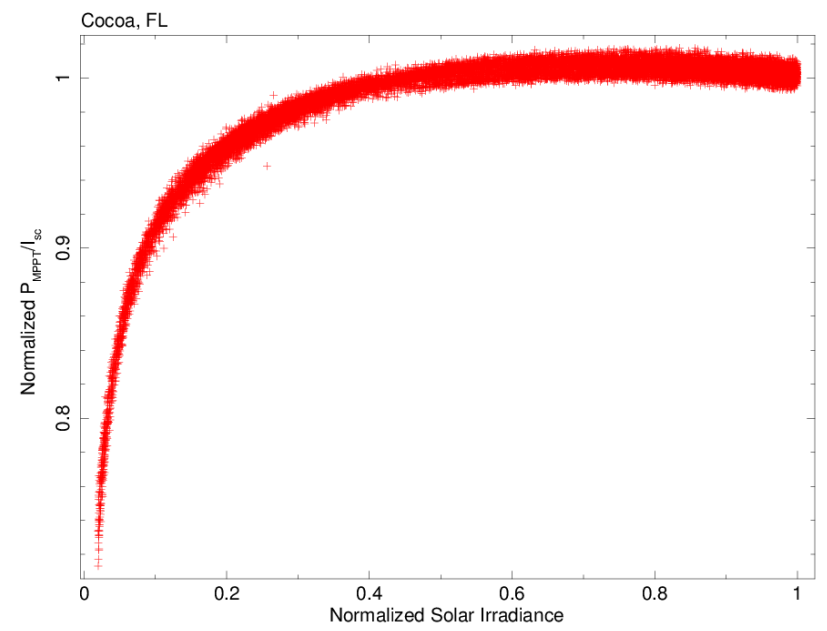

Fig. 1. The ratio of normalized power to normalized short circuit current is related to the efficiency of the module. Data are from a single crystalline module during its deployment at Cocoa, FL.

It is also known that at low light levels photovoltaic efficiency is reduced in a nonlinear way. The effect of the shunt resistance becomes important since the total resistance of the module during operation approaches the value of the shunt resistance. The percentage loss of power due to the shunt resistance increases at low solar irradiances. In order to study the effect of the change of the efficiency of a module as a function of solar irradiance stainless steel meshes of various transmittances were constructed. These meshes when used in various combinations allow us to vary the incoming solar irradiance by a factor of 10 . The backside module temperature is recorded along with the solar irradiance with and without the mesh. The unobstructed solar irradiance in front of the mesh is used to assess the stability of the ambient conditions. The resulting data can be used to model the performance of modules of the same technology, e.g. single or multi crystalline, amorphous $\mathrm{Si}$, etc. Another simplified approach utilizes good quality IV curves of a module under different solar irradiances as in the NREL data. It relies on the fact that the short circuit current under typical conditions is not affected by the shunt resistance and that the power is affected by air mass and angle of incidence in the same way as the short circuit current. The selected data satisfy the following conditions: air mass less than 2, angle of incidence less than 60 degrees and global irradiance less than $1000 \mathrm{~W} / \mathrm{m}^{2}$. The instantaneous maximum power and short circuit current are corrected for temperature and normalized to their STC values. Subsequently, the two quantities are divided to form a ratio independent of airmass and angular effects. The ratio is plotted as a function of normalized incoming solar irradiance as in Figure 1.

\section{VALIDATION DATA}

Currently, the model takes into account the solar irradiance, the cell temperature, the angle of incidence, and the low irradiance performance. Runs are conducted with and without the air mass correction which to a first approximation is assumed to be that of the short circuit current. High quality validation data are required to check the performance of the model. NREL carried out a field campaign at three different sites in the United States and with several PV modules of different technologies at different climatic conditions [5]. Quality assessment methods were implemented by NREL to create a reliable data base that can be used by scientists for validation and performance purposes. The regular maintenance of the experiment along with the quality of the measurement equipment provided specific measurement errors.

In this work data acquired from a single-crystalline silicon module are processed. The module was first deployed at a tilt of $28^{\circ} .5$ and an azimuth of $180^{\circ}$ in Cocoa, FL (subtropical climate) for more than one year. During this time I-V traces were recorded every five minutes along with several environmental parameters. The same module was then deployed at Eugene, OR (marine climate) at a tilt of $44^{\circ}$ and an azimuth of $180^{\circ}$. NREL performed indoor performance measurements at Standard Test Conditions (STC) of the deployed modules. The adopted STC power used in the calculations is 83 Watts. The data from the two different sites mentioned above offer an excellent tool to study the effects of diverse climates upon the performance of a module.

\section{DISCUSSION}

A first version of the model has been presented in [3] along with the mathematical expressions describing the various factors that affect the photovoltaic power. As already mentioned the model can be improved if diffuse and beam solar irradiance data are available. The model adopts the spectral function that describes the effects of the propagation of sunlight through the atmosphere upon the short circuit current as given in [4] and [6]. A fourth degree polynomial is determined through measurements of the short circuit current at various air masses during a day. It is noted that actual atmospheric conditions are not stable during the day leading to differences before and after solar noon. Formally, maximum power point measurements should be conducted but these should be corrected for the low irradiance losses. A separate function refers to the effect caused by the fact that light does not enter the module at normal incidence. A generic curve normalized to unity at zero angle of incidence can be adopted from $[4,6]$ or separate measurements can be carried out using the module under study. The model also takes into account the reduction in efficiency of a cell or module when operating under solar irradiances significantly less than that of the standard test conditions. The influence of other effects can be included in the model if their functions are known. Seasonal dust effects can be included to the model if related data are available from independent sources. A fixed factor may be 
used to account for the aging of the modules. If the module is new, this factor can be set to 1 .

NREL provides error estimates for all the data available from this experiment. The relative errors on the MPPT photovoltaic power and the solar irradiance, among other parameters, were calculated. In the current analysis the selected data are characterized by relative errors of less than $2 \%$ in both PV power and solar irradiance. Two data sets were created for the model validation, one for each of the sites with experimental data adopting the constraint of $2 \%$. The data set from Eugene, OR contains 21552 different measurements, while the data set from Cocoa, FL contains 24776 measurements.

The ratio between model and measured or experimental data is calculated as $\mathrm{P}_{\text {model }} / \mathrm{P}_{\text {data }}$, while the percent difference between the model and the measured data is calculated as $\left(\mathrm{P}_{\text {model }}-\mathrm{P}_{\text {data }}\right) / \mathrm{P}_{\text {data. }}$. Histograms of the percentage difference are created and fit with Gaussian profiles to determine the sigma of the distribution. Figure 2 shows the histogram of the percentage difference between modeled and observed values of PV power for Cocoa, FL. Figure 3 shows the corresponding plot for Eugene, OR. A Gaussian fit to the Cocoa, FL histogram results in a sigma of 0.012, while the sigma for the Eugene, OR data is 0.016 . The one sigma limit is accompanied by a possibility of $68 \%$ which is not large enough to confidently limit a parameter. Adopting the three sigma confidence limit, the typical uncertainty of the model is around 0.05 or $5 \%$. Examination of the actual power produced by the module and that estimated by the model shows a linear relation with the scatter increasing at the lower values of the power (Figure 4).

A number of reasons may explain this behavior. The variation of efficiency as a function of solar irradiance should be determined by laboratory measurements at predetermined conditions. However, such detailed data are not available in the data sheet. Usually, the reduction in efficiency at a level of e.g. $200 \mathrm{~W} / \mathrm{m}^{2}$ is quoted by the manufacturers. The air mass effect is more complicated than the polynomial expression adopted in the calculations and its unique dependence upon the elevation of the sun. Precipitable water and the aerosol content of the atmosphere are expected to affect the performance of a module. Note also that the atmospheric conditions are not stable during the day but this effect cannot be modeled in the absence of detailed data as is generally the case, e.g. a grid connected photovoltaic power facility. In addition, the air mass effect is less important in single or multi crystalline modules because their spectral response is less affected by the scattering of light in the atmosphere. Amorphous Si or CdTe modules are more affected due to their bluer response. The NREL data include modules of different technologies including CdTe, CIGS, a-Si which will be strongly affected by the spectral distribution of light during low sun elevation angles. Different functions will be required to account for the actual implications of the atmospheric conditions upon the power production. A simple model using a small number of input parameters is validated against quality data from an experiment carried out at different climatic conditions in USA. The current analysis suggests an accuracy of the model of around 5\%. The full analysis of all module technologies will provide an even better assessment of its performance.

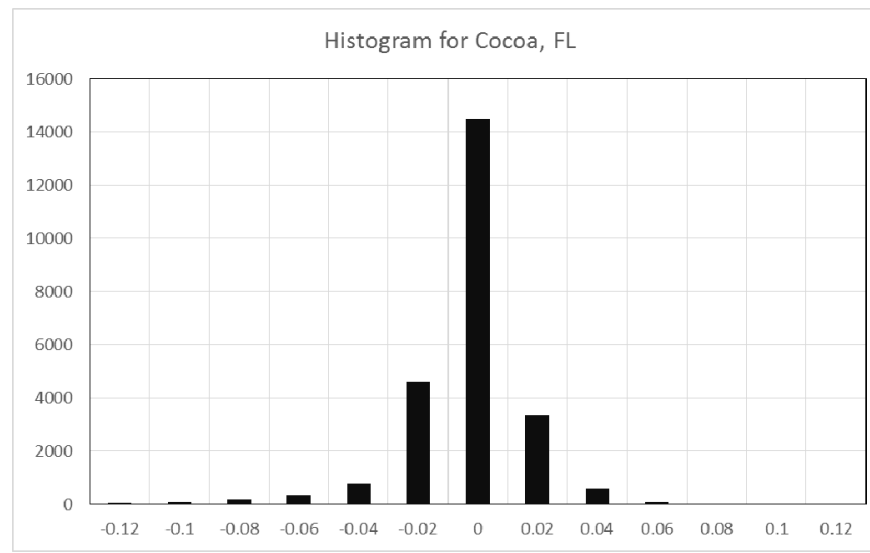

Fig. 2. The histogram of the percentage difference between modeled and measured photovoltaic power for Cocoa, FL.

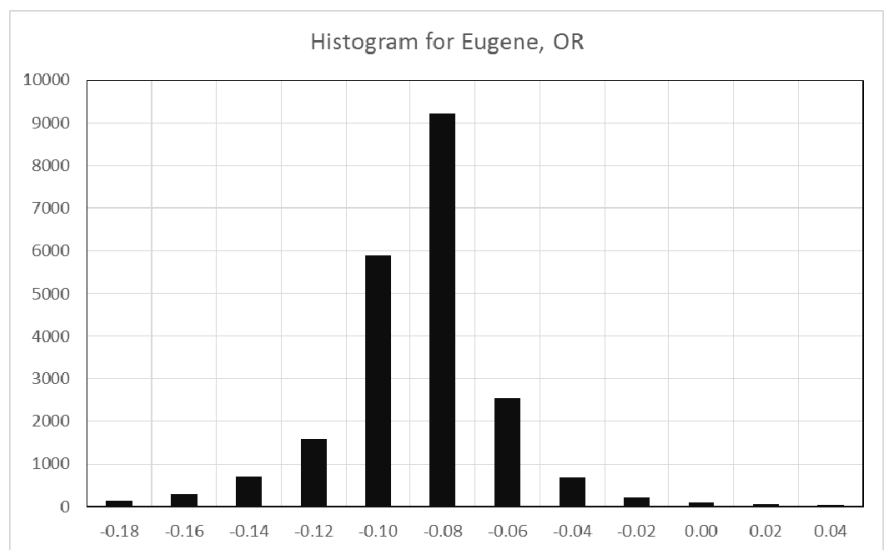

Fig. 3. The histogram of the percentage difference between modeled and measured photovoltaic power for Eugene, OR.

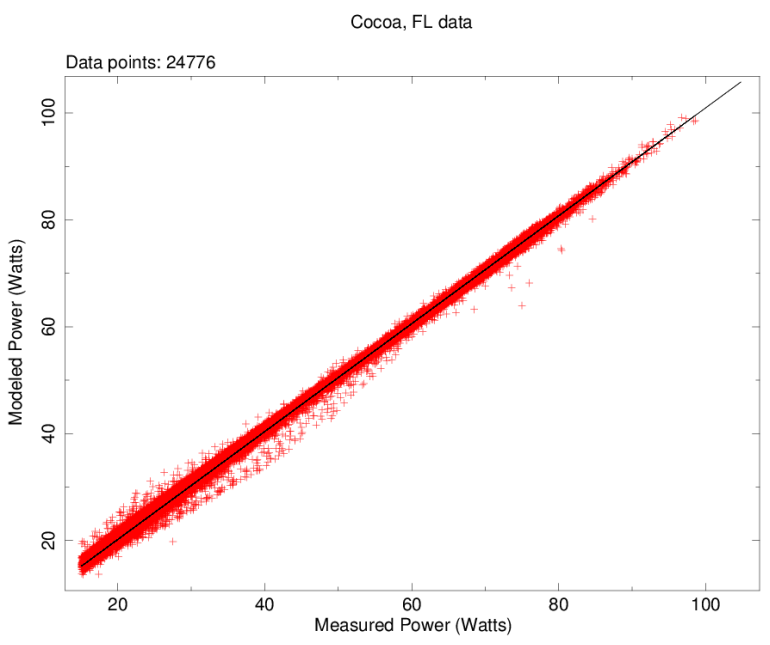

Fig. 4. A one to one plot of the measured and modeled photovoltaic power from the single crystalline module under consideration. 


\section{ACKNOWLEDGMENT}

This project is implemented through the Operational Program "Education and Lifelong Learning", Action Archimedes III and is co-financed by the European Union (European Social Fund) and Greek national funds (National Strategic Reference Framework 2007 - 2013). The authors would also to thank B. Marion for providing the NREL Test's facility data. The Eugene data used in this project was generated under the NREL subcontract number AFU-2-220721 .

\section{REFERENCES}

[1] K. Ding, Z. Ye, T. Reindl, "Comparison of parameterisation Models for the Estimation of the maximum power output of pv modules", Energy Procedia, Vol. 25, pp. 101-107, 2012
[2] F. Mavromatakis, G. Makrides, G. Georghiou, A. Pothrakis, Y. Franghiadakis, E. Drakakis, E. Koudoumas, "Modelling the photovoltaic potential of a site", Renewable Energy, Vol. 35, pp. 1387-1390, 2010

[3] F. Mavromatakis, F. Vignola, Y. Franghiadakis, "Modelling Photovoltaic power", 10th International Conference on Deregulated Electricity Market Issues in South Eastern Europe (DEMSEE), Budapest, Hungary, 24-25 September, 2015

[4] D. L. King, J. A. Kratochvil, W. E. Boyson, "Measuring Solar Spectral and Angle-of-Incidence Effects on Photovoltaic Modules and Solar Irradiance Sensors", 26th IEEE Photovoltaic Specialists Conference, Anaheim, California, USA, September 29 - October 3, 1997

[5] W. Marion, A. Anderberg, C. Deline, S. Glick, M. Muller, G. Perrin, J. Rodriguez, S. Rummel, K. Terwilliger, T. J. Silverman, User's manual for Data for validating models for pv module performance, National Renewable Energy Laboratoy, Technical Report, VTP-5200-61610, 2014

[6] A. H. Fanney, M. W. Davis, B. P. Dougherty, D. L. King, W. E. Boyson, J. A. Kratochvil, "Comparison of Photovoltaic Module Performance Measurements", Transactions of the ASME, Vol. 128, pp. 152-160. 2006 